\title{
Cytotoxic Steroids From The Stembak of Chisocheton celebicus KOORD
}

\author{
Dewa Gede Katja ${ }^{1, *}$, Desi Harneti ${ }^{2}$, Tri Mayanti ${ }^{2}$, Nurlelasari ${ }^{2}$, Rani Maharani ${ }^{2,3}$ \\ Yoshihito Shiono ${ }^{4}$, Unang Supratman ${ }^{2,3}$ \\ ${ }^{1}$ Department of Chemistry, Faculty of Mathematics and Natural Sciences, Sam Ratulangi University \\ Kampus Kleak, Manado, 95115, North Sulawesi, Indonesia \\ ${ }^{2}$ Department of Chemistry, Faculty of Mathematics and Natural Sciences, Padjadjaran University \\ Jatinangor 45363, Indonesia \\ ${ }^{3}$ Central Laboratory, Universitas Padjadjaran, Jatinangor 45363, Indonesia \\ ${ }^{4}$ Department of Food, Life, and Environmental Science, Faculty of Agriculture, Yamagata University \\ Tsuruoka, Yamagata 997-8555, Japan \\ *Corresponding author: dewakatja@yahoo.com
}

Received: August 2019; Revision: October 2019; Accepted: November 2019; Available online: November 2019

\begin{abstract}
In the course of our continuing search for anticancer compounds from Chisocheton species, three steroids,

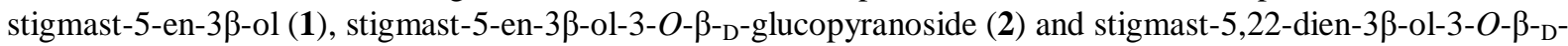
glucopyranoside $(\mathbf{3})$, were obtained from the stembark of Chisocheton celebicus. The structures of compound 13 were identified with spectroscopic data including IR, 1D-NMR, 2D-NMR and TOF-MS, as well as by comparing with those spectral data previously. Compounds 1-3, were evaluated for their cytotoxic effects against $\mathrm{P}-388$ murine leukemia cells and displayed the cytotoxicity activity with $\mathrm{IC}_{50}$ values of $12.45 \pm 0.050,52.27 \pm$ 0.031 and $62.52 \pm 0.076 \mu \mathrm{g} / \mathrm{mL}$, respectively.
\end{abstract}

Keyword: Chisocheton celebicus koord, cytotoxic activity, Meliaceae, P-388 murine leukemia cells, steroid.

\begin{abstract}
Abstrak
Sebagai bagian dari studi kami tentang senyawa kandidat antikanker dari tumbuhan Chisocheton Indonesia, tiga senyawa steroid, stigmast-5-en-3 $\beta$-ol (1), stigmast-5-en-3 $\beta$-ol-3- $O-\beta{ }_{-}{ }^{-}$glucopyranoside (2) dan stigmast-5,22dien-3 $\beta$-ol-3-O- $\beta{ }_{-}{ }_{\mathrm{D}}$-glucopyranoside (3), telah diperoleh dari kulit batang Chisocheton celebicus. Struktur kimia senyawa 1-3 diidentifikasikan berdasarkan data spektroskopi meliputi, IR, NMR-1D, NMR-2D dan TOF-MS serta perbandingan dengan data spektra sebelumnya. Senyawa 1-3 diuji efek sitotoksiknya terhadap sel murin leukemia P-388 dan memperlihatkan aktivitas sitotoksik dengan nilai $\mathrm{IC}_{50}$ berturut-turut, $5.14 \pm 0.015,30.12 \pm$ 0.0025 dan $35.86 \pm 0.053 \mu \mathrm{M}$.
\end{abstract}

Kata kunci: Aktivitas sitotoksik, Chisocheton celebicus koord, Meliaceae, sel murin leukimia P-388, steroid.

DOI: $10.15408 / j k v . v 5 i 2.12132$

\section{INTRODUCTION}

The genus Chisocheton, belong to family Meliaceae, consisting more than 50 plants and distributes in Nepal, India, Myanmar, China, Thailand, Indonesia, Malaysia, and Papua New Guinea (Vossen and Umali, 2002). Previous investigation of chemical constituents on Chisocheton plants had reported contain sesquiterpenoids (Phongmaykin et al., 2008), dammarane-type triterpenoids (Phongmaykin et al., 2008; Inada et al., 1993), lanostane-type triterpenoid (Katja et al., 2017a), tirucallane-type triterpenoids (Zhang et al., 2012) apo-tirucallane-type triterpenoids (Zhang et al., 2012; Yang et al., 2011), euphane-type triterpenoids (Supratman et al., 2019); limonoids (Maneerat et al., 2008; Laphookhieo et al., 2008; Mohamad et al., 2009; Yang et al., 2009; Najmuldeen et al., 2011; Wong et al., 2011; Nurlelasari et al., 2017; Supriatno et al., 2018), steroids 
(Najmuldeen et al., 2011) and phenolics (Inada et al., 1993).

As part of our investigation on cytotoxic compounds from Chisocheton plants, we reported a mexicanolide-type limonoid from $C$. macrophyllus (Nurlelasari et al., 2017), a trijugin-type limonoid and lanostane-type triterpenoid from C. cumingianus (Katja et al., 2017a; Katja et al., 2017b), vilacinine-type limonoid from C. pentandrus (Supriatno et al., 2018) and euphane type-triterpenoid from $C$. patens Blume (Supratman et al., 2019). In the our continuing search for cytotoxic compounds from Indonesia Chisocheton plants, we found that the $n$-hexane and ethyl acetate extracts of the stembark of $C$. celebicus exhibited a cytotoxic activity against P-388 murine leukemia cells with $\mathrm{IC}_{50}$ of $20.72 \pm 0.02$ and $18.48 \pm 0.03 \mu \mathrm{g} / \mathrm{mL}$, respectively. In this paper, the isolation and structural identification of three steroids along with their cytotoxic activity against P-388 murine leukimia cells are discribed.

\section{MATERIALS AND METHODS Experimental Procedure}

Melting points were obtained on an electrothermal melting point instrument. The infrared spectra and mass spectra were obtained on a SHIMADZU IR Prestige-21 in $\mathrm{KBr}$ and Waters Xevo QTOF-MS, respectively. The NMR data was recorded using a JEOL ECZ-500 at $500 \mathrm{MHz}$ for ${ }^{1} \mathrm{H}$ and $125 \mathrm{MHz}$ for ${ }^{13} \mathrm{C}$, using tetramethylsilane as an internal standard. Column chromatography was carried out on the silica gel 60 (70-230 and 230-400 mesh), after which TLC analysis was carried out on $60 \mathrm{GF}_{254}(0.25 \mathrm{~mm})$ using various solvent systems, spots was detected by spraying with $10 \%$ sulfuric acid in ethanol followed by heating.

\section{Plant Material}

The stembarks of C. celebicus were obtained in Bogor Botanical Garden, West Java Province, Indonesia in April 2012. The plant was determined by Mr. Ismail at the Bogoriense Herbarium, Bogor, Indonesia and deposited at the herbarium with the number of Bo-1305316.

\section{Determination of Cytotoxic Activities}

The P388 cells were growth into 96-well plates at an initial cell density of approximately $3 \times 10^{4}$ cells $\mathrm{cm}^{-3}$. After 24 hours of incubation for cell attachment and growth, several concentrations of samples were added. The samples added were first dissolved in dimethyl sulfoxide at the required concentration as a negative control. Subsequent six desirable concentrations were prepared using phosphoric buffer solution, $\mathrm{pH}$ $=$ 7.30-7.65). Control wells received only dimethyl sulfoxide. The assay was terminated after a 48 hours incubation period by adding MTT reagent [3-(4,5-dimethylthiazol-2-yl)2,5-diphenyl tetrazolium bromide; also named as thiazol blue] and the incubation was continued for another four hours, in which the MTT-stop solution containing sodium dodecyl sulphate was added and another 24 hours incubation was conducted. Optical density was read by using a micro plate reader at $550 \mathrm{~nm}$. $\mathrm{IC}_{50}$ values were taken from the plotted graph of percentage live cells compared to control (\%), receiving only phosphoric buffer solution, $\mathrm{pH}=7.30-7.65$ and dimethyl sulfoxide, versus the tested concentration of compounds $(\mu \mathrm{g} / \mathrm{mL})$. The $\mathrm{IC}_{50}$ value is the concentration required for 50\% growth inhibition. Each assay and analysis was run in triplicate and averaged.

\section{Extraction and Isolation}

The dried stembark $(1.5 \mathrm{~kg})$ was soaked in methanol $(12 \mathrm{~L})$ for 3 days. After evaporate of the methanol on the rotary evaporator, the concentrated of $\mathrm{MeOH}$ extract (120.5 g) was dissolved in $\mathrm{H}_{2} \mathrm{O}$ and then partitioned successively with $n$-hexane, EtOAc, and $n$ $\mathrm{BuOH}$. Evaporation on the rotary evaporator produced the crude extracts of $n$-hexane (20.3 g), EtOAc (10.4 g), and $n$-BuOH (11.6 g), respectively. The $n$-hexane extract $(20.3 \mathrm{~g})$ was separated by vacuum liquid chromatography on silica gel 60 by using $n$ hexane and ethyl acetate as a gradien eluent to give nine fractions (A-I). Fraction A (6 g) was separated by column chromatographed on silica gel with a $n$-hexane- $\mathrm{CH}_{2} \mathrm{Cl}_{2}$ as a gradient eluen (10:0-1:1) to give ten subfractions (A01-A10). Subfraction A03 was further separated by column chromatographed on silica gel with $n$-hexane: $\mathrm{CHCl}_{3}(9: 1)$ as an eleunt to give $1(14.5 \mathrm{mg})$. The EtOAc extract $(12.4 \mathrm{~g})$ was separated by column chromatography on silica gel using a $n$-hexane and ethyl acetate as an eleunt to give eight fractions $(\mathrm{J}-\mathrm{Q})$. Fraction K $(927.6 \mathrm{mg})$ was column chromatographed on silica gel, eluted with a $n$-hexane-EtOAc (10:0-0:10) as eluent 
to give 2 (16.3 $\mathrm{mg}$ ). Fractions $\mathrm{P}$ (3.86 g) was column chromatographed on silica gel, eluted with a $\mathrm{CHCl}_{3}-\mathrm{Me}_{2} \mathrm{CO}$ as a eleunt $(10: 0-4: 1)$ to give $3(5.5 \mathrm{mg})$.

\section{RESULTS AND DISCUSSION}

The $n$-hexane and EtOAc fraction were separated by several column chromatography followed by cytotoxic test to produce three cytotoxic steroids 1-3 (Figure $1)$.

Stigmast-5-en-3ß-ol (1), white crystals, m.p. $134-136{ }^{\circ} \mathrm{C}$; IR (KBr) $v_{\max } 3430$, 2950, 2860,1460,1360, 1240, $1060 \mathrm{~cm}^{-1}$; ${ }^{1} \mathrm{H}-$ NMR $\left(\mathrm{CDCl}_{3}, 500 \mathrm{MHz}\right)$ and ${ }^{13} \mathrm{C}-\mathrm{NMR}$ $\left(\mathrm{CDCl}_{3}, 125 \mathrm{MHz}\right)$, see Table 1; TOFMS (negative ion mode) $\mathrm{m} / \mathrm{z} 413.0811[\mathrm{M}-\mathrm{H}]^{-}$, (calcd. $\mathrm{C}_{29} \mathrm{H}_{49} \mathrm{O}^{-}, m / z$ 413.3789).

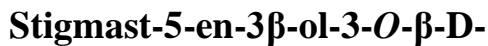

glucopyranoside (4), white amorphous powder; m.p. (decomposed); IR (KBr) $v_{\max }$ 3433, 1639, 1461, 1380, $1053 \mathrm{~cm}^{-1}$; ${ }^{1} \mathrm{H}-\mathrm{NMR}$ (pyridine- $d_{5}, \quad 500 \mathrm{MHz}$ ) and ${ }^{13} \mathrm{C}-\mathrm{NMR}$ (pyridine- $d_{5}, 125 \mathrm{MHz}$ ), see Table 1 .

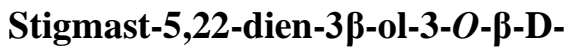

glucopyranoside (5), white amorphous powder; m.p. (decomposed); IR (KBr) $v_{\max }$ 3450, 1630, 1445, 1370, $1050 \mathrm{~cm}^{-1}$; ${ }^{1} \mathrm{H}-\mathrm{NMR}$ (pyridine- $\left.d_{5}, 500 \mathrm{MHz}\right)$ and ${ }^{13} \mathrm{C}-\mathrm{NMR}\left(\mathrm{CDCl}_{3}\right.$, $125 \mathrm{MHz}$ ), see Table 1.

Compound 1. The HR-TOFMS spectrum of compound 1 showed molecular ion at $\mathrm{m} / \mathrm{z} 413.0811$ (calcd. $\mathrm{m} / \mathrm{z}$ 413.3789), which consistent to the molecular formula of $\mathrm{C}_{29} \mathrm{H}_{50} \mathrm{O}$ and thus requiring hydrogen deficiency index of five, consisting of one pairs of $\mathrm{C} \mathrm{sp}^{2}$ and tetracyclic stigmastane-type steroid. The infra red spectra displayed the presence of hydroxyl $\left(3430 \mathrm{~cm}^{-1}\right)$ alipahtics (2950 and $\left.2860 \mathrm{~cm}^{-1}\right)$, olefinic (1460 cm-1), gem-dimethyl $\left(1360\right.$ and $\left.1240 \mathrm{~cm}^{-1}\right)$ and ether group $\left(1060 \mathrm{~cm}^{-1}\right)$.

The ${ }^{1} \mathrm{H}-\mathrm{NMR}$ spectrum displayed two tertiary methyl at $\delta_{\mathrm{H}} 1.00(\mathrm{Me}-18)$ and 0.68 (Me-19), three secondary methyl at $\delta_{\mathrm{H}} 0.92$ $(3 \mathrm{H}, \mathrm{d}, J=6.2 \mathrm{~Hz}, \mathrm{Me}-21), 0.83(3 \mathrm{H}, \mathrm{d}, J=$ $6.5 \mathrm{~Hz}, \mathrm{Me}-26)$, and $0.81(\mathrm{~d}, J=5.2 \mathrm{~Hz}, \mathrm{Me}-$ 27 ), one primary methyl group at $\delta_{\mathrm{H}} 0.84(\mathrm{t}, J$ $=5.2 \mathrm{~Hz}, \mathrm{Me}-29)$, corresponding to stigmastane-type steroid (Cayme and Ragasa, 2004; Farabi et al., 2017). An oxygenated $\mathrm{sp}^{2}$ methine at $\delta_{\mathrm{H}} 5.35(\mathrm{~d}, J=5.2 \mathrm{~Hz}, \mathrm{H}-6)$ and oxygenated $\mathrm{sp}^{3}$ methine at $\delta_{\mathrm{H}} 3.52(1 \mathrm{H}, \mathrm{m}, \mathrm{H}-$ $3)$, were identified at ${ }^{1} \mathrm{H}$ NMR spectra. The vicinal proton was also confirmed by the ${ }^{1} \mathrm{H}$ ${ }^{1} \mathrm{H}$ Correlated Spectroscopy (COSY) spectrum (Figure 2). ${ }^{1} \mathrm{H}-{ }^{1} \mathrm{H}$ COSY countour was identified at $\mathrm{C}_{2}-\mathrm{C}_{3}-\mathrm{C}_{4}$ suggested that position of a secondary alcohol at $\mathrm{C}-3$. The countour was also observed at $\mathrm{C}_{6}-\mathrm{C}_{7}-\mathrm{C}_{8}$, indicated that the position of double bond at $\mathrm{C}_{5}-\mathrm{C}_{6}\left(\Delta^{5,6}\right)$. The ${ }^{13} \mathrm{C}-\mathrm{NMR}\left(\mathrm{CDCl}_{3} 125 \mathrm{MHz}\right)$ and heteronuclear single quantum coherence (HSQC) and Distortionless enhancement by polarization transfer (DEPT) spectra displayed the presence of six methyl, an olefinic methine, an olefinic quartenary carbon, and a oxygenated methine at $\delta_{\mathrm{C}} 72.0(\mathrm{C}-3)$, suggested the presence of stigmastane-type steroid (Cayme and Ragasa, 2004; Farabi et al., 2017). These unsaturations were determined for one of total hydrogen deficiency index of five. The remaining four degrees of hydrogen deficiency index were corresponding to stigmastane-type steroid. A detail analysis cof the NMR data of $\mathbf{1}$ with to those of $\beta$-sitosterol (Chaturvedula and Prakash, 2012; Farabi et al., 2017), indicated that the structure of both compounds displed highly similarity, therefore compound $\mathbf{1}$ was identified as a stigmast-5-en-3 $\beta$-ol ( $\beta$ sitosterol).

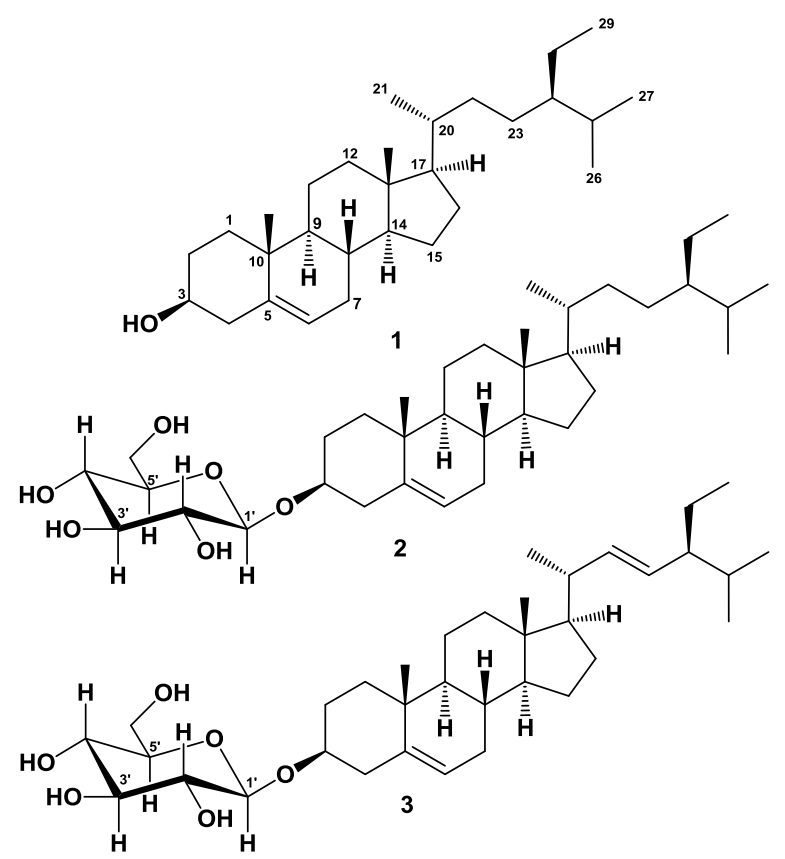

Figure 1. Chemical structure of compounds 1-3.

Compound 2, the molecular formula of 2 was identified as $\mathrm{C}_{35} \mathrm{H}_{60} \mathrm{O}_{6}$, from NMR data (Table 1). The infra red spectrum showed absorption band at 3460, 2890, 1650, 1475 
and 1380, and $1070 \mathrm{~cm}^{-1}$, respectively, corresponding to hydroxyl, aliphatics, gemdimethyl, olefinic and ether groups. A NMR spectra of $\mathbf{2}$ similar to those of $\mathbf{1}$, except the presence of sugar moiety in $\mathbf{2}$. The signals of oxygenated methylene at $\delta_{\mathrm{H}} 4.27\left(2 \mathrm{H}, \mathrm{m}, \mathrm{H}-6^{\prime}\right)$ and an anomeric signal proton at $\delta_{\mathrm{H}} 4.53(1 \mathrm{H}$, d, $J=7.5 \mathrm{~Hz}, \mathrm{H}^{-1}{ }^{\prime}$ ), as well as of four oxygenated methines at $\delta_{\mathrm{H}} 4.25(1 \mathrm{H}, \mathrm{dd}, J=$ $\left.5.5,7.5 \mathrm{~Hz}, \mathrm{H}-2^{\prime}\right), 3.95(1 \mathrm{H}, \mathrm{dd}, J=5.5,7.2$
$\left.\mathrm{Hz}, \mathrm{H}-3^{\prime}\right), 4.03(1 \mathrm{H}, \mathrm{dd}, J=74.5,7.2 \mathrm{~Hz}, \mathrm{H}-$ $\left.4^{\prime}\right)$, and $4.39\left(1 \mathrm{H}, \mathrm{d}, J=4.5 \mathrm{~Hz}, \mathrm{H}-5^{\prime}\right)$, supporting for a glucose moiety. The ${ }^{13} \mathrm{C}$ NMR signal of anomeric carbon located at $\delta_{\mathrm{C}} 102.4$ $\left(\mathrm{C}-1^{\prime}\right)$, suggesting the $\beta$-glucose. In comparison of $\mathbf{2}$ with literature data (Harneti et al., 2014; Farabi et al., 2017), showed good agreement, therefore compound $\mathbf{2}$ was identified as stigmast-5-en-3 $\beta$-ol-3-O- $\beta$-Dglucopyranoside ( $\beta$-sitosterol glucoside).

Table 1. NMR data for compounds 1-3.

\begin{tabular}{|c|c|c|c|c|c|c|}
\hline \multirow{2}{*}{$\mathbf{C}$} & \multicolumn{2}{|r|}{1} & \multicolumn{2}{|r|}{2} & \multicolumn{2}{|r|}{3} \\
\hline & $\delta_{\mathrm{C}}$ (mult.) & $\delta_{\mathrm{H}}(\Sigma \mathrm{H}$, mult., $J(\mathrm{~Hz})$ & $\delta_{\mathrm{C}}$ (mult.) & $\delta_{\mathrm{H}}(\Sigma \mathrm{H}$, mult., $J(\mathrm{~Hz})$ & $\delta_{\mathrm{C}}$ (mult.) & $\delta_{\mathrm{H}}(\Sigma \mathrm{H}$, mult., $J(\mathrm{~Hz})$ \\
\hline 1 & $37.4(\mathrm{t})$ & $1.68(1 \mathrm{H}, \mathrm{m})$ & $37.3(t)$ & $1.83(1 \mathrm{H}, \mathrm{m})$ & $37.3(\mathrm{t})$ & $1.83(1 \mathrm{H}, \mathrm{m})$ \\
\hline & & $1.70(1 \mathrm{H}, \mathrm{dd}, 2.4,4.5)$ & & $1.73(1 \mathrm{H}, \mathrm{dd}, 1.2,8.5)$ & & $1.90(1 \mathrm{H}, \mathrm{dd}, 3.4,7.8)$ \\
\hline 2 & $31.8(\mathrm{t})$ & $1.50(1 \mathrm{H}, \mathrm{m})$ & $31.9(\mathrm{t})$ & $1.90(1 \mathrm{H}, \mathrm{dd}, 3.0,9.0)$ & $31.9(\mathrm{t})$ & $1.90(1 \mathrm{H}, \mathrm{dt}, 1.2,9.4)$ \\
\hline & & $1.60(1 \mathrm{H}, \mathrm{m})$ & & $2.42(1 \mathrm{H}, \mathrm{t}, 10.8)$ & & $2.42(1 \mathrm{H}, \mathrm{dd}, 2.3,9.4)$ \\
\hline 3 & $72.0(\mathrm{~d})$ & $3.52(1 \mathrm{H}, \mathrm{m})$ & 78.5 (d) & $3.90(1 \mathrm{H}, \mathrm{m})$ & $78.5(d)$ & $3.90(1 \mathrm{H}, \mathrm{m})$ \\
\hline 4 & $42.3(\mathrm{t})$ & $\begin{array}{l}2.23(1 \mathrm{H}, \mathrm{dd}, 3.4,8.5) \\
2.45(1 \mathrm{H}, \mathrm{m})\end{array}$ & $39.2(\mathrm{t})$ & $\begin{array}{l}2.06(1 \mathrm{H}, \mathrm{dd}, 1.8,12.8) \\
2.68(1 \mathrm{H}, \mathrm{dd}, 1.8,9.4)\end{array}$ & $39.2(\mathrm{t})$ & $\begin{array}{l}1.66(1 \mathrm{H}, \mathrm{dd}, 1.2,9.7) \\
2.67(1 \mathrm{H}, \mathrm{m})\end{array}$ \\
\hline 5 & $140.9(\mathrm{~s})$ & - & $140.7(\mathrm{~s})$ & - & $140.7(\mathrm{~s})$ & - \\
\hline 6 & $121.9(\mathrm{~d})$ & $5.35(1 \mathrm{H}, \mathrm{t}, 5.2)$ & $121.8(\mathrm{~d})$ & $5.28(1 \mathrm{H}, \mathrm{d}, 4.8)$ & $121.8(\mathrm{~d})$ & $5.28(1 \mathrm{H}, \mathrm{d}, 3.6)$ \\
\hline 7 & $32.1(\mathrm{t})$ & $\begin{array}{l}1.99(1 \mathrm{H}, \mathrm{dd}, 5.2,8.5) \\
2.01(1 \mathrm{H}, \mathrm{m})\end{array}$ & $29.2(\mathrm{t})$ & $\begin{array}{l}1.76(1 \mathrm{H}, \mathrm{m}) \\
1.80(1 \mathrm{H}, \mathrm{dd}, 1.2,4.8)\end{array}$ & $29.3(\mathrm{t})$ & $\begin{array}{l}1.80(1 \mathrm{H}, \mathrm{m}) \\
1.84(1 \mathrm{H}, \mathrm{dd}, 4.2,8.2)\end{array}$ \\
\hline 8 & $32.1(\mathrm{~d})$ & $0.85(1 \mathrm{H}, \mathrm{dd}, 4.5,8.5)$ & $32.0(\mathrm{~d})$ & $1.69(1 \mathrm{H}, \mathrm{m})$ & $32.0(\mathrm{~d})$ & $1.69(1 \mathrm{H}, \mathrm{m})$ \\
\hline 9 & $50.3(\mathrm{~d})$ & $1.43(1 \mathrm{H}, \mathrm{dd}, 8.5,9.8)$ & $50.1(\mathrm{~d})$ & $0.96(1 \mathrm{H}, \mathrm{t}, 7.1)$ & $50.2(\mathrm{~d})$ & $1.00(1 \mathrm{H}, \mathrm{d}, 9.6)$ \\
\hline 10 & $36.7(\mathrm{~s})$ & - & $36.7(\mathrm{~s})$ & - & $36.7(\mathrm{~s})$ & - \\
\hline 11 & $21.3(\mathrm{t})$ & $\begin{array}{l}1.46(1 \mathrm{H}, \mathrm{m}) \\
1.55(1 \mathrm{H}, \mathrm{dd}, 4.8,9.2)\end{array}$ & $21.1(\mathrm{t})$ & $\begin{array}{l}1.47(1 \mathrm{H}, \mathrm{m}) \\
1.68(1 \mathrm{H}, \mathrm{m})\end{array}$ & $21.1(\mathrm{t})$ & $\begin{array}{l}1.47(1 \mathrm{H}, \mathrm{m}) \\
1.52(1 \mathrm{H}, \mathrm{m})\end{array}$ \\
\hline 12 & $39.9(t)$ & $\begin{array}{l}1.23(1 \mathrm{H}, \mathrm{m}) \\
1.35(1 \mathrm{H}, \mathrm{dd}, 2.5,9.2)\end{array}$ & $39.7(t)$ & $\begin{array}{l}1.30(1 \mathrm{H}, \mathrm{m}) \\
1.50(1 \mathrm{H}, \mathrm{dd}, 4.5,9.8)\end{array}$ & $39.8(\mathrm{t})$ & $\begin{array}{l}1.43(1 \mathrm{H}, \mathrm{dd}, 3.0,9.5) \\
1.32(1 \mathrm{H}, \mathrm{m})\end{array}$ \\
\hline 13 & $42.4(\mathrm{~s})$ & - & $42.3(\mathrm{~s})$ & - & $42.3(\mathrm{~s})$ & - \\
\hline 14 & 56.9 (d) & $0.95(1 \mathrm{H}, \mathrm{m})$ & $56.6(d)$ & $1.04(1 \mathrm{H}, \mathrm{dd}, 4.5,7.6)$ & $56.7(d)$ & $1.05(1 \mathrm{H}, \mathrm{m})$ \\
\hline 15 & $26.2(\mathrm{t})$ & $\begin{array}{l}1.58(1 \mathrm{H}, \mathrm{dd}, 4.5,9.8) \\
1.66(1 \mathrm{H}, \mathrm{m})\end{array}$ & $24.3(\mathrm{t})$ & $\begin{array}{l}1.49(1 \mathrm{H}, \mathrm{m}) \\
1.54(1 \mathrm{H}, \mathrm{m})\end{array}$ & $24.4(\mathrm{t})$ & $\begin{array}{l}1.48(1 \mathrm{H}, \mathrm{m}) \\
1.50(1 \mathrm{H}, \mathrm{dd}, 6.8,9.6)\end{array}$ \\
\hline 16 & $28.4(\mathrm{t})$ & $\begin{array}{l}1.16(1 \mathrm{H}, \mathrm{m}) \\
1.30(1 \mathrm{H}, \mathrm{m})\end{array}$ & $28.4(t)$ & $\begin{array}{l}1.53(1 \mathrm{H}, \mathrm{m}) \\
1.62(1 \mathrm{H}, \mathrm{m})\end{array}$ & $28.4(\mathrm{t})$ & $\begin{array}{l}1.66(1 \mathrm{H}, \mathrm{m}) \\
1.72(1 \mathrm{H}, \mathrm{m})\end{array}$ \\
\hline 17 & $56.2(\mathrm{~d})$ & $1.10(1 \mathrm{H}, \mathrm{m})$ & $56.0(\mathrm{~d})$ & $1.20(1 \mathrm{H}, \mathrm{dd}, 9.5,11.4)$ & $56.1(\mathrm{~d})$ & $1.19(1 \mathrm{H}, \mathrm{m})$ \\
\hline 18 & $12.0(\mathrm{q})$ & $1.00(3 \mathrm{H}, \mathrm{s})$ & $11.8(\mathrm{q})$ & $0.58(3 \mathrm{H}, \mathrm{s})$ & $11.8(\mathrm{q})$ & $0.59(3 \mathrm{H}, \mathrm{s})$ \\
\hline 19 & $19.0(\mathrm{q})$ & $0.68(3 \mathrm{H}, \mathrm{s})$ & $19.8(\mathrm{q})$ & $0.86(3 \mathrm{H}, \mathrm{s})$ & $19.8(\mathrm{q})$ & $0.86(3 \mathrm{H}, \mathrm{s})$ \\
\hline 20 & $36.3(\mathrm{~d})$ & $1.86(1 \mathrm{H}, \mathrm{dd}, 2.5,6.2)$ & 36.2 (d) & $1.36(1 \mathrm{H}, \mathrm{m})$ & $36.2(d)$ & $1.36(1 \mathrm{H}, \mathrm{m})$ \\
\hline 21 & $19.2(q)$ & $0.92(3 \mathrm{H}, \mathrm{d}, 6.2)$ & $19.0(q)$ & $0.91(3 \mathrm{H}, \mathrm{d}, 6.0)$ & $19.0(\mathrm{q})$ & $0.92(3 \mathrm{H}, \mathrm{d}, 4.2)$ \\
\hline 22 & $34.1(\mathrm{t})$ & $1.15(2 \mathrm{H}, \mathrm{m})$ & $34.0(\mathrm{t})$ & $1.17(2 \mathrm{H}, \mathrm{m})$ & $138.7(\mathrm{~d})$ & $4.98(1 \mathrm{H}, \mathrm{dd}, 7.8,8.4)$ \\
\hline 23 & $26.2(\mathrm{t})$ & $1.23(2 \mathrm{H}, \mathrm{m})$ & $26.1(\mathrm{t})$ & $1.29(2 \mathrm{H}, \mathrm{m})$ & $129.3(\mathrm{~d})$ & $5.13(1 \mathrm{H}, \mathrm{dd}, 7.8,8.4)$ \\
\hline 24 & 45.9 (d) & $1.52(1 \mathrm{H}, \mathrm{dd}, 1.6,5.6)$ & $45.8(\mathrm{~d})$ & $1.01(1 \mathrm{H}, \mathrm{dd}, 3.4,7.8)$ & $45.8(\mathrm{~d})$ & $1.01(1 \mathrm{H}, \mathrm{dd}, 4.6,8.3)$ \\
\hline 25 & $29.3(d)$ & $1.60(1 \mathrm{H}, \mathrm{dd}, 1.2,7.8)$ & 30.1 (d) & $1.59(1 \mathrm{H}, \mathrm{dd}, 2.1,6.5)$ & $30.1(\mathrm{~d})$ & $1.65(1 \mathrm{H}, \mathrm{m})$ \\
\hline 26 & $19.6(q)$ & $0.83(3 \mathrm{H}, \mathrm{d}, 6.5)$ & $19.3(\mathrm{q})$ & $0.80(3 \mathrm{H}, \mathrm{d}, 5.0)$ & $19.3(\mathrm{q})$ & $0.81(3 \mathrm{H}, \mathrm{d}, 4.9)$ \\
\hline 27 & $20.0(\mathrm{q})$ & $0.79(3 \mathrm{H}, \mathrm{d}, 5.2)$ & $18.8(\mathrm{q})$ & $0.78(3 \mathrm{H}, \mathrm{d}, 5.0)$ & $18.8(\mathrm{q})$ & $0.79(3 \mathrm{H}, \mathrm{d}, 4.9)$ \\
\hline 28 & $23.2(\mathrm{t})$ & $1.30(2 \mathrm{H}, \mathrm{m})$ & $23.2(\mathrm{t})$ & $1.34(2 \mathrm{H}, \mathrm{m})$ & $23.2(\mathrm{t})$ & $\begin{array}{l}1.25(1 \mathrm{H}, \mathrm{m}) \\
1.32(1 \mathrm{H}, \mathrm{dd}, 2.4,7.4)\end{array}$ \\
\hline 29 & $12.2(\mathrm{q})$ & $0.83(3 \mathrm{H}, \mathrm{t}, 5.2)$ & $12.0(\mathrm{q})$ & $0.83(3 \mathrm{H}, \mathrm{t}, 2.5)$ & $12.0(\mathrm{t})$ & $0.83(3 \mathrm{H}, \mathrm{t}, 2.4)$ \\
\hline $1^{\prime}$ & & & $102.4(\mathrm{~d})$ & $4.53(1 \mathrm{H}, \mathrm{d}, 7.5)$ & $102.4(\mathrm{~d})$ & $4.52(1 \mathrm{H}, \mathrm{dd}, 2.4,8.5)$ \\
\hline $2^{\prime}$ & & & $75.2(\mathrm{~d})$ & $4.25(1 \mathrm{H}, \mathrm{dd}, 5.5,7.5)$ & $75.2(d)$ & $4.22(1 \mathrm{H}, \mathrm{dd}, 8.5,9.3)$ \\
\hline $3^{\prime}$ & & & $77.7(\mathrm{~d})$ & $3.95(1 \mathrm{H}, \mathrm{dd}, 5.5,7.2)$ & $77.7(d)$ & $3.94(1 \mathrm{H}, \mathrm{dd}, 7.3,9.3)$ \\
\hline $4^{\prime}$ & & & $78.4(\mathrm{~d})$ & $4.07(1 \mathrm{H}, \mathrm{dd}, 4.5,7.2)$ & $78.4(\mathrm{~d})$ & $4.02(1 \mathrm{H}, \mathrm{dd}, 7.3,7.5)$ \\
\hline $5^{\prime}$ & & & $71.5(\mathrm{~d})$ & $4.39(1 \mathrm{H}, \mathrm{d}, 4.5)$ & $71.5(\mathrm{~d})$ & $4.37(1 \mathrm{H}, \mathrm{dd}, 7.5,7.7)$ \\
\hline $6^{\prime}$ & & & $62.6(t)$ & $4.27(2 \mathrm{H}, \mathrm{m})$ & $62.6(\mathrm{t})$ & $4.25(2 \mathrm{H}, \mathrm{m})$ \\
\hline
\end{tabular}

*Measured in $\mathrm{CDCl}_{3}\left(500 \mathrm{MHz}\right.$ for ${ }^{1} \mathrm{H}$ and $125 \mathrm{MHz}$ for $\left.{ }^{13} \mathrm{C}\right)$ 
Compound 3, the molecular formula was determined to be $\mathrm{C}_{35} \mathrm{H}_{58} \mathrm{O}_{6}$ from NMR data (Table 1). The IR spectrum showed absorption peak at 3475, 2890, 1630, 1445 and 1370 and $1050 \mathrm{~cm}^{-1}$, respectively, corresponding to hydroxyl, aliphatic, olefinic, gem-dimethyl and ether groups. NMR spectra of $\mathbf{3}$ very similar with 2 , except the presence of an additional double bond at $\delta_{\mathrm{H}} 4.98$ and 5.13 (each $1 \mathrm{H}, \mathrm{dd}, J=7.8,8.4 \mathrm{~Hz}, \mathrm{H}-22, \mathrm{H}-23$ ) and $\delta_{\mathrm{C}} 138.7(\mathrm{C}-22)$ and $129.3(\mathrm{C}-23)$ in $3 . \mathrm{In}$ comparison of $\mathbf{3}$ with literature data (Harneti et al., 2014; Farabi et al., 2017), showed good agreement, therefore compound $\mathbf{3}$ was identified as stigmast-5,22-dien-3 $\beta-\mathrm{ol}-3-O-\beta-\mathrm{D}^{-}$ glucopyranoside (stigmasterol glucoside).

The cytotoxic assay was conducted as mentioned in the previous papers (Alley et al., 1988; Hakim et al., 2007; Supratman et al., 2019) and was used an artonin $\mathrm{E}\left(\mathrm{IC}_{50} 0.75\right.$ $\mu \mathrm{g} / \mathrm{mL}$ ) as a positive control (Hakim et al., $2007)$. The cytotoxic activity of stigmast-5-en$3 \beta$-ol (1) stronger than stigmast-5-en-3 $\beta$-ol-3$O-\beta$-D-glucopyranoside (2) and stigmast-5,22dien-3 $\beta$-ol-3- $O-\beta$-D-glucopyranoside indicated that the presence of sugar group can decrease cytotoxic activity in steroid structure.

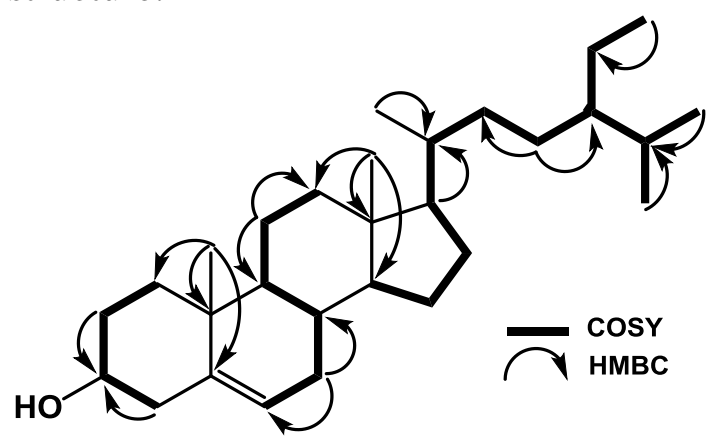

Figure 2. Selected COSY and HMBC correlation for compound 1

\section{CONCLUSION}

Three cytotoxic steroids were investigated from the bark of $C$. celebicus and identified as stigmast-5-en-3 $\beta$-ol (1), stigmast5-en-3 $\beta$-ol-3- $O$ - $\beta$-D-glucopyranoside (2), and stigmast-5,22-dien-3 $\beta$-ol-3- $O-\beta$-D-

glucopyranoside (3). The presence of sugar moities can decrease cytotoxic activity. The investigation of these steroids were shown in this species for the first time.

\section{ACKNOWLEDGMENTS}

This research was supported by Kemristekdikti, Indonesia (Hibah Penelitian Dasar Perguruan Tinggi, 2018-2019, by Dewa Gede Katja). We thank Dr. Ahmad Darmawan, M.Si and Mrs. Sofa Fajriah, M.Si in the Research Center for Chemistry, Indonesian Science Institute, for NMR measurements.

\section{REFERENCES}

Alley MC, Scudiero DA, Monks A, Hursey ML, Czerwinski MJ, Fine DL, Abbott BJ, Mayo JG, Shoemaker RH, Boyd MR. 1988. Feasibility of drug screening with panels of tumor cell lines using a microculturetetrazolium assay. Cancer Research. 48: 589-601.

Cayme J, Ragasa C. 2004. Structure elucidation of $\beta$-stigmasterol and $\beta$-sitosterol from Sesbania grandiflaora (Linn). Pers. and $\beta$ carotene from Heliotropium indicum Linn by NMR spectroscopy. J. Kimika. 20: 512.

Chaturvedula VSP, Prakash I. 2012. Isolation of stigmasterol and $\beta$-sitosterol from the dichloromethane extract of Rubus suavissimus. Int. Curr. Pharm. J. 1: 239242.

Farabi K, Harneti D, Nurlelasari, Maharani R, Hidayat AC, Supratman U, Awang K, Shiono Y. 2017. Cytotoxic steroids from the bark of aglaia argentea (Meliaceae). CMU J. Nat. Sci. 16(4): 293-306.

Hakim EH, Achmad SA, Juliawaty LD, Makmur L, Syah YM, Aimi A, Kitajima M, Takayama H, Ghisalberti EL. 2007. Prenylated flavonoids and related compounds of the Indonesian Artocarpus (Moraceae). J. of Nat. Med. 61(2): 229-236.

Harneti D, Supriadin A, Ulfah M, Safari A, Supratman U, Awang K, Hayashi H. 2014. Cytotoxic constituents from the bark of Aglaia eximia (Meliaceae). Phytochem. Lett. 8: 28-31.

Inada A, Sukemawa M, Murata H, Nakanishi T, Tokuda H, Nishino H, Iwashima, Darnaedi DJ, Murata J. 1993. Phytochemical studies on Maleaceous plant. part VIII. structures and inhibitory effects on Epstein-Barr virus activation of triterpenoid from leaves of Chisocheton macrophyllus King. Chem. Pharm. Bull. 41(3): 617-619. 
Katja DG, Farabi K, Nurlelasari, Harneti D, Mayanti T, Supratman U, Awang K, Hayashi H. 2017a. Cytototoxic constituents from the bark of Chisocheton cumingianus (Meliaceae). Journal of Asian Natural Products Research. 6: 1-5.

Katja DG, Farabi K, Nurlelasari, Harneti D, Maharani R, Julaeha E, Hidayat AT, Mayanti T, Supratman U. 2017b. cytotoxic steroids from the stem bark of chisocheton cumingianus (Meliaceae). Molekul. 12(1): 1-7.

Laphookhieo S, Maneerat W, Koysomboon S, Kiattansakul R, Chantrapromma K, Syers JK. 2008. A novel limonoid from the seeds of Chisocheton siamensis. Can. J. Chem. 86: 205-208.

Maneerat W, Laphoohiero S, Koysomboon S, Chantrapromma K. 2008. Antimalarial, antimycobacterial and cytotoxic limonoid from Chisocheton siamensis. Phytomedicine. 15: 1130-1134..

Mohamad K, Hirasawa Y, Litaudon M, Awang K, Hamid A, Takeya K, Ekasari W, Widyawaruyanti A, Zaini NC, Morita H. 2009. Ceramicines B-D, new antiplasmodial limonoids from Chisocheton ceramicus. Bioorganic \& Medicinal Chemistry. 17: 727-730.

Najmuldeen IA, Hadi AHA, Awang K, Mohamad K, Ketuly KA, Mukhtar MR, Chong SL, Chan G, Nafiah MA, Weng NS, Shirota O, Hosoya T, Nugroho A, Morita H. 2011. Chisomicines A-C, limonoids from Chisocheton ceramicus. J. Nat. Prod. 74: 1313-1317.

Nurlelasari, Katja DG, Harneti D, Wardayo MM, Supratman U, Awang K. 2017. limonoids from the seeds of Chisocheton macrophyllus. Chemistry of Natural Compounds: 53(1): 83-87.

Phongmaykin J, Kumamoto T, Ishikawa T, Suttisri R, Saifah E. 2008. A New sesquiterpene and other terpenoid constituents of Chisocheton penduliflorus. Arch Pharm Res. 31: 21-27.

Supriatno, Nurlelasari, Herlina T, Harneti D, Maharani R, Hidayat AT, Mayanti T, Supratman U, Azmi MN, Shiono Y. 2018. A new limonoid from stem bark of Chisocheton pentandrus (Meliaceae). Natural Products Research. 1: 1-6.

Supratman U, Naibaho W, Salam S, Maharani R, Hidayat AT, Harneti D, Nurlelasari, Shiono Y. 2019. Cytotoxic triterpenoids from the bark of Chisocheton patens Blume (Meliaceae). Phytochemistry Letters. 30: 81-87.

Vossen VD, Umali BE. (Editors). 2002. Plant resources of south-east Asia no. 14 vegetable oils and fats, Prosea Foundation, Bogor, Indonesia. 150.

Yang MH, Wang JG, Luo JG, Wang XB, Kong LY. 2011. Chisopanins A-K, 11 new protolimonoids from Chisocheton paniculatus and their anti-inflammatory activities. Bioorganic \& Medicinal Chemistry. 19: 1409-1417.

Yang MH, Wang JS, Luo JG, Wang XB, Kong LY. 2009. Tetranortriterpenoids from Chisocheton Paniculatus. J. Nat. Prod. 70: 1532-1532.

Wong CP, Shimada M, Nagakura Y, Nugroho AE, Hirasawa Y, Kaneda T, Awang K, Hamid A, Hadi A, Mohamad K, Shio M, Morita H. 2011. Ceramicines E-I, new limonoids from Chisocheton ceramicus. Chem. Farm. Bull. 59: 407-411.

Zhang F, Feng HE, Bin W, Sheningg C, Mian Y. 2012. New apotirucallane type triterpenoid from Chisocheton paniculatus. Nat. Prod. Bioprospect. 2: 235-239. 